\title{
Circularly Polarized Light Generated by Plasmon Antenna for All-Optical Magnetic Recording
}

\author{
K. Tamura, T. Ota, Y. Ashizawa*, A. Tsukamoto*, \\ A. Itoh", S. Ohnuki*, and K. Nakagawa* \\ Graduate School of Science and Technology, Nihon University, 7-24-1 Narashinodai, Funabashi, 274-8501, Japan \\ ${ }^{*}$ College of Science and Technology, Nihon University, 7-24-1 Narashinodai, Funabashi, 274-8501, Japan
}

All-optical magnetic recording by applying circularly polarized light is a promising new technology for ultra-fast speed recording. This technique can control the direction of magnetization without any applied magnetic field. It is, however, unsuitable for creating small magnetic domains for high density recording, because optical spot size is restricted to the diffraction limit. Previously, we proposed methods to generate a localized circularly polarized light by localized surface plasmon antennas (LSPA), and to utilize a surface plasmon polariton waveguide (SPPW) for high efficiency in energy propagation. This study investigates a new antenna array that combines plasmon cross antenna and high efficient SPPW. The circular polarization is evaluated by the degree of circularity $C$ and power intensity of electric field $I$. The circularly polarized light of $5 \mathrm{~nm}$ in diameter is located at the center of the LSPA, which keeps the $C$ value at 0.8 . It is revealed that the confined circular polarization can be generated by the LSPA with SPPW.

Key words: all-optical magnetic recording, localized circularly polarized light, localized surface plasmon antenna, surface plasmon polariton, surface plasmon polariton waveguide

\section{Introduction}

All-optical magnetic recording with circularly polarized light has been proposed for high-speed recording because the magnetization can be reversed by a trigger laser pulse of $40 \mathrm{fs}$ circularly polarized light without any applied magnetic field ${ }^{1}$. It is, however, unsuitable for creating small magnetic domains on the order of ten $\mathrm{nm}$, which is necessary for high density magnetic recording. One of the methods which has been proposed to resolve this problem is using a localized surface plasmon antenna (LSPA) to generate a confined circularly polarized light ${ }^{2-4}$. Besides, a system using surface plasmon polariton waveguide ${ }^{5)}$ (SPPW) was also investigated to achieve a high efficiency in energy propagation. It is indispensable both to confine a circularly polarized light and to achieve a high efficiency in energy propagation. In this paper, we study an energy efficient system to localize circularly polarized light by using LSPA with SPPW. We evaluate the degree of circularity $C$ and power intensity of electric field $I^{6)}$ as the confined circularly polarized light. It was accomplished that the circular polarization was confined to $5 \mathrm{~nm}$ in diameter, of which the value of $C$ was kept at 0.8 .

\section{Fundamental evaluation of a plasmon antenna without SPPW}

Before evaluating a combined system between LSPA and SPPW, we first wanted to know if a circularly polarized light could be created by LSPA on a metal sheet with a liner polarized light source without the
SPPW. We calculated this as a fundamental evaluation model using only LSPA placed on the bottom of a $\mathrm{Au}$ sheet, as shown in Fig. 1. Calculation was done by the Finite-Difference Time-Domain (FDTD) method.

\subsection{LSPA computational model}

A computational model is shown in Fig. 1. Au antennas were placed on the bottom surface of a $\mathrm{Au}$ sheet of size and thickness at $300 \times 300 \mathrm{~nm}^{2}$ and $95 \mathrm{~nm}$. The lengths of the antennas were $30 \mathrm{~nm}$ and $70 \mathrm{~nm}$, each at a thickness of $35 \mathrm{~nm}$. Electromagnetic wave was calculated by the FDTD method. The calculation area

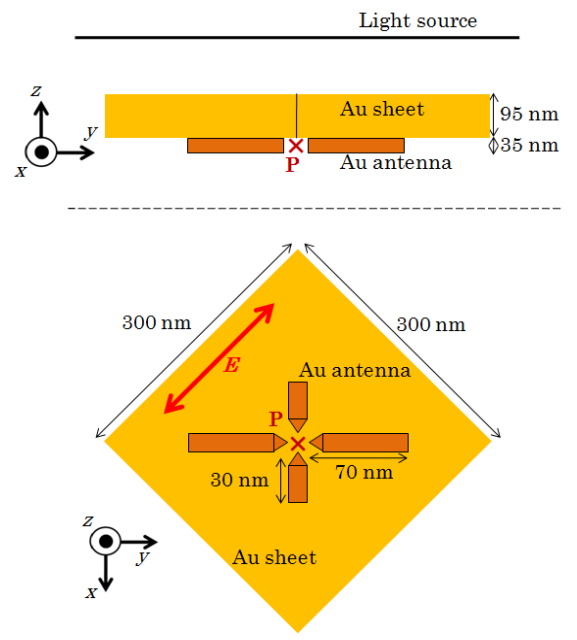

Fig. 1 Computational model for fundamental evaluation. Au cross antenna was placed on a $\mathrm{Au}$ sheet. Light source projected on the top surface of the $\mathrm{Au}$ sheet. Electric field was observed at point $\mathrm{P}$. 
was $2,000 \times 2,000 \times 2,000 \mathrm{~nm}^{3}$ and the minimum cell size was $0.5 \times 0.5 \times 0.5 \mathrm{~nm}^{3}$ with Mur's absorption boundaries condition ${ }^{7)}$. A Gaussian linear polarized light with $780 \mathrm{~nm}$ of the wavelength impinged on the top surface of the $\mathrm{Au}$ sheet, as shown in Fig. 1. The light source diameter, the peak electric field intensity, and the Gaussian diameter $\left(1 / \mathrm{e}^{2}\right)$ of the light were $1,560 \mathrm{~nm}$, $1 \mathrm{~V} / \mathrm{m}$, and $780 \mathrm{~nm}$, respectively. The incident light propagated along the $z$ axis.

\subsection{Evaluation of circularly polarized light}

The electric near field at the point $\mathrm{P}$ generated by the LSPA was studied, as shown in Fig. 1. Each component of the electric field $\left(E_{\mathrm{x}}, E_{\mathrm{y}}\right.$ and $\left.E_{\mathrm{z}}\right)$ as a function of time is shown in Fig. 2. After $30 \mathrm{fs}$, the phase difference between $E_{\mathrm{x}}$ and $E_{\mathrm{y}}$ was nearly 90 degrees, with both intensities at almost the same value. The small intensity difference can be adjusted by changing the lengths of antennas ${ }^{4}$. The proposed near field antenna array can create a confined circularly polarized

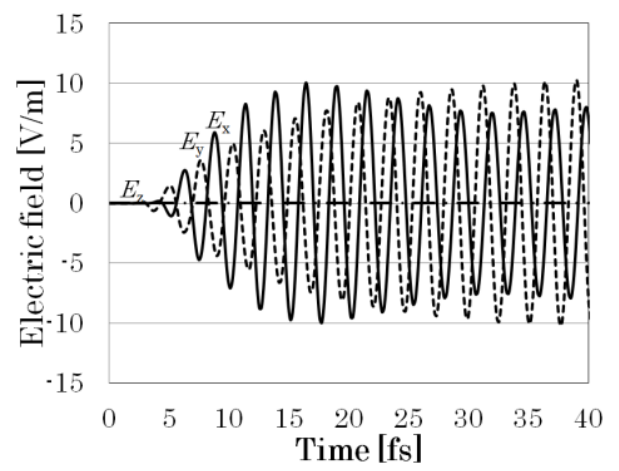

Fig. 2 Time responses of electric field $x, y$ and $z$ components $\left(E_{\mathrm{x}}, E_{\mathrm{y}}\right.$ and $\left.E_{\mathrm{z}}\right)$ at the center of the cross antenna. light from a linear polarized light. This result means that an expected high efficient energy propagation effect by SPPW would turn out when the LSPA receives indirect energy from the SPPW, as in past research ${ }^{5)}$.

\section{Individual control of a phase ( $E_{\mathrm{x}}$ and $\left.E_{\mathrm{y}}\right)$}

When we generate circularly polarized light created by LSPA placed on the bottom of a SPPW, it is crucial to control the two orthogonal components of electric field phase. Therefore, the effect of antenna length as well as position on a phase control of the electric field was studied.

\subsection{Computational model for rectangular shaped plasmon antenna with SPPW}

This computational model was composed of LSPA and SPPW, as shown in Fig. 3. The dielectric wave path was made of $\mathrm{Ta}_{2} \mathrm{O}_{5}$. The SPPW made of Au was placed along the $\mathrm{Ta}_{2} \mathrm{O}_{5}$ optical path. The thickness of the $\mathrm{Au}$ SPPW and the distance between the optical path and the SPPW was $180 \mathrm{~nm}$ and $10 \mathrm{~nm}$, respectively. The shape of the SPPW is shown in Fig. 3 (b). The thickness was uniform, but the width $W$ decreases in the $z$ direction. The $W$ of the top half of the SPPW was 3,000 nm, but in the bottom half the $W$ decreases as the sides taper together. This tapering was for leading energy to the bottom of the SPPW. The rectangular shaped plasmon antenna was attached on the bottom of the SPPW, and covered with $\mathrm{Al}_{2} \mathrm{O}_{3}$. Two types of LSPA, which are shown in Figs. 3 (c) and (d), were respectively designed to create an electric field of $x$ and $y$ components. A laser light with $780 \mathrm{~nm}$ of wavelength provided a linear polarized light (transverse magnetic wave). The electric field intensity had a Gaussian distribution with $1 \mathrm{~V} / \mathrm{m}$ at the center and $780 \mathrm{~nm}$ in the diameter of the optical spot

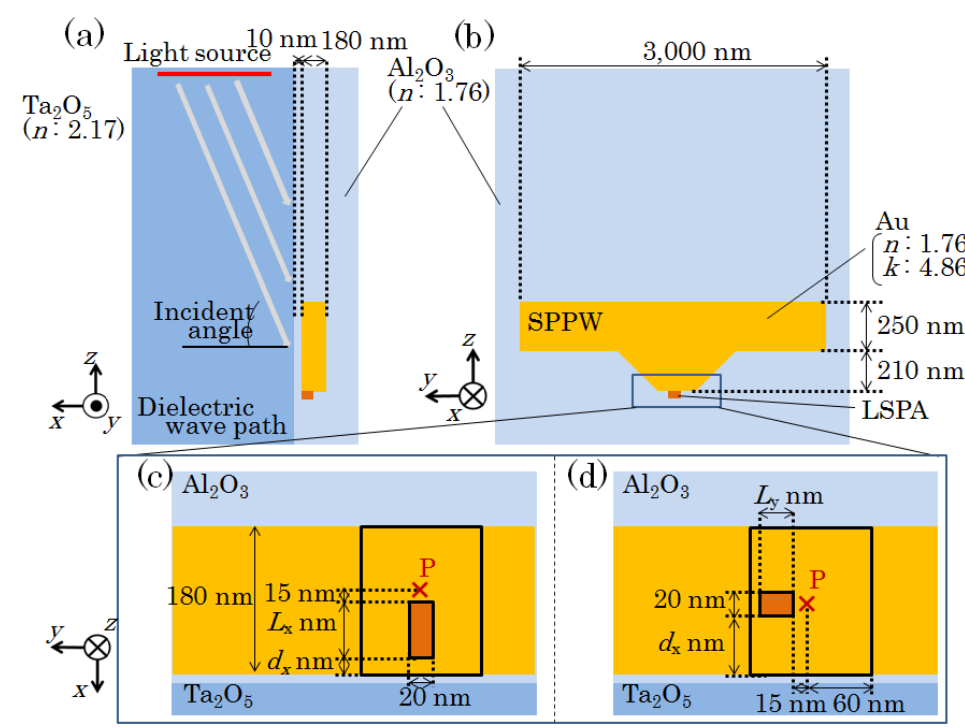

(a) Side view

(b) Front view

(c), (d)

Bottom view

Fig. 3 Computational models to study the effect of antenna length and position on a phase control of electric field. These models were composed of dielectric waveguide, SPPW and LSPA. The types of LSPAs shown as (c) and (d) at bottom views were respectively designed to create an electric field $x$ and $y$ components. 
$\left(1 / \mathrm{e}^{2}\right)$. The incident angle was 80 degrees, which was set to meet the condition of the total reflection. The electric field generated by rectangular shaped plasmon antenna was observed at point $\mathrm{P}$, as shown in Fig. 3 .

\subsection{The phase evaluation of $E_{\mathrm{x}}$ and $E_{\mathrm{y}}$}

Time responses were investigated for $x$ and $y$ components of electric field $\left(E_{\mathrm{x}}\right.$ and $\left.E_{\mathrm{y}}\right)$ in terms of antenna length and position. $d_{\mathrm{x}}$ was defined as the distance from the edges of SPPW and LSPA as shown in Fig. $3\left(\right.$ c) and (d). $L_{\mathrm{x}}$ and $L_{\mathrm{y}}$ were antenna lengths in $x$ direction and $y$ direction respectively. The $E_{\mathrm{x}}$ and $E_{\mathrm{y}}$ as a function of time are shown in Figs. 4 and 5, respectively. The rectangular shaped LSPA, to create electric field $x$ or $y$ components, was designed as follows: $d_{\mathrm{x}}=0 \mathrm{~nm}$ and $L_{\mathrm{x}}=60 \mathrm{~nm}(\# 1), d_{\mathrm{x}}=0 \mathrm{~nm}$ and $L_{\mathrm{x}}=175 \mathrm{~nm}(\# 2), d_{\mathrm{x}}=110$ $\mathrm{nm}$ and $L_{\mathrm{x}}=60 \mathrm{~nm}(\# 3), d_{\mathrm{x}}=60 \mathrm{~nm}$ and $L_{\mathrm{y}}=15 \mathrm{~nm}(\# 4)$, $d_{\mathrm{x}}=60 \mathrm{~nm}$ and $L_{\mathrm{y}}=45 \mathrm{~nm}(\# 5), d_{\mathrm{x}}=150 \mathrm{~nm}$ and $L_{\mathrm{y}}=15$ $\mathrm{nm}$ (\#6). The $E_{\mathrm{x}}$ 's phase was delayed at 51 degrees when the length of $L_{\mathrm{x}}$ changed from 60 to $150 \mathrm{~nm}$, or the $d_{\mathrm{x}}$ changed from 0 to $110 \mathrm{~nm}$. On the other hand, the $E_{\mathrm{y}}$ 's phase was shifted +21 or -21 degrees when the $L_{\mathrm{y}}$ changed from 15 to $45 \mathrm{~nm}$, or the $d_{\mathrm{x}}$ changed from 60 to $150 \mathrm{~nm}$. It was revealed that we could control the phase of $E_{\mathrm{x}}$ and $E_{\mathrm{y}}$ by changing the length and position of the LSPA, which was attached on the bottom of the SPPW.

\section{Circular polarization by LSPA with SPPW}

\subsection{Computational model for circular polarization generated by LSPA with SPPW}

It was revealed that the phase of orthogonal electric field was controllable by altering the shape and the location of LSPA. The combination system between LSPA and SPPW was studied by applying rectangular shaped antennas, but it was not easy to create circular polarization. Therefore, we study SPPW with an asymmetric cross antenna, as explained in section 2 . The computational model is shown in Fig. 6. The asymmetric cross antenna was placed on the bottom of the SPPW. The antenna was set close to the dielectric waveguide to generate high-intensity near field because almost all the energy in the form of surface plasmon polariton was delivered by the dielectric waveguide's side of SPPW. The lengths of the cross antenna were $50 \mathrm{~nm}$ and $30 \mathrm{~nm}$ and these widths were $20 \mathrm{~nm}$. The thickness of antenna projected from the bottom surface of SPPW was $35 \mathrm{~nm}$. The SPPW was the same structure shown in Fig. 3.

\subsection{The evaluation of circularly polarized light generated by LSPA with SPPW}

The electric field at the center of the cross antenna was calculated. Fig. 7 shows each component of the electric field $\left(E_{\mathrm{x}}, E_{\mathrm{y}}\right.$ and $\left.E_{\mathrm{z}}\right)$ as a function of time. After 70 fs from laser irradiation, the electric field amplitudes of the $x$ and $y$ components were almost the same value

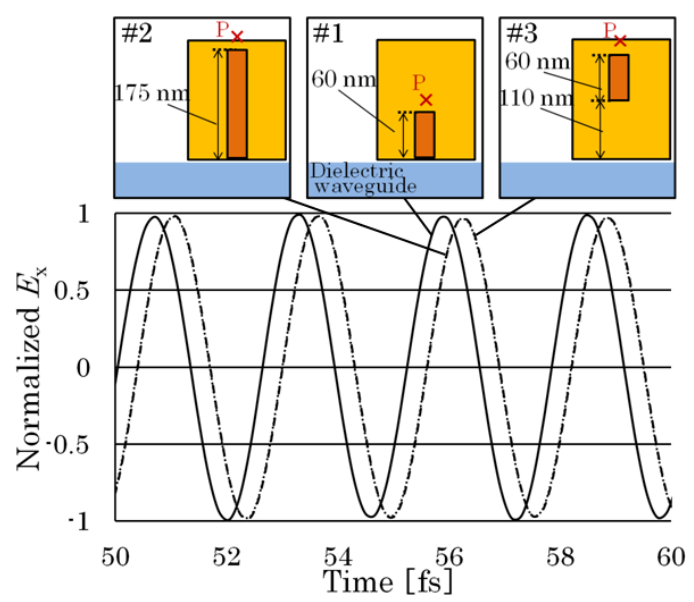

Fig. 4 Time responses of normalized electric field $x$ component. $d_{\mathrm{x}}$ and $L_{\mathrm{x}}$ of \#1, \#2 and \#3 were (0 $\mathrm{nm}, 60 \mathrm{~nm}),(0 \mathrm{~nm}, 175 \mathrm{~nm})$ and $(110 \mathrm{~nm}, 60$ $\mathrm{nm})$, respectively.

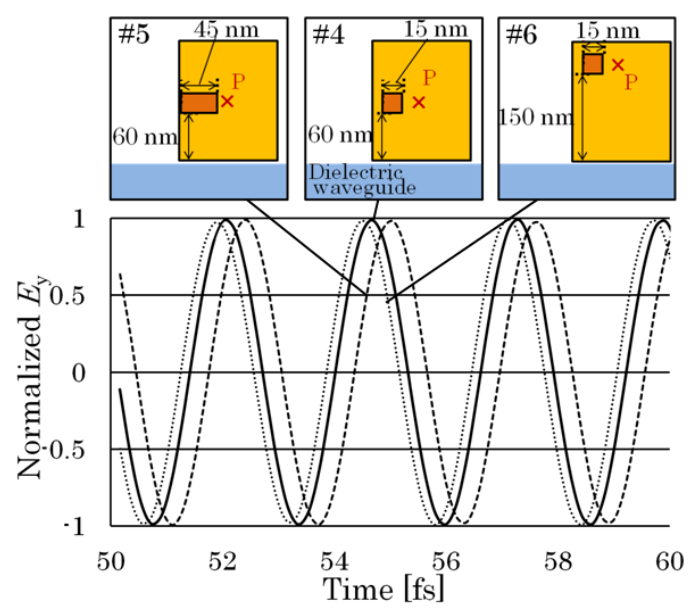

Fig. 5 Time responses of normalized electric field $y$ component. $d_{\mathrm{x}}$ and $L_{\mathrm{y}}$ of \#4, \#5 and \#6 were (60 $\mathrm{nm}, 15 \mathrm{~nm}),(60 \mathrm{~nm}, 45 \mathrm{~nm})$ and $(150 \mathrm{~nm}, 15$ $\mathrm{nm})$, respectively.

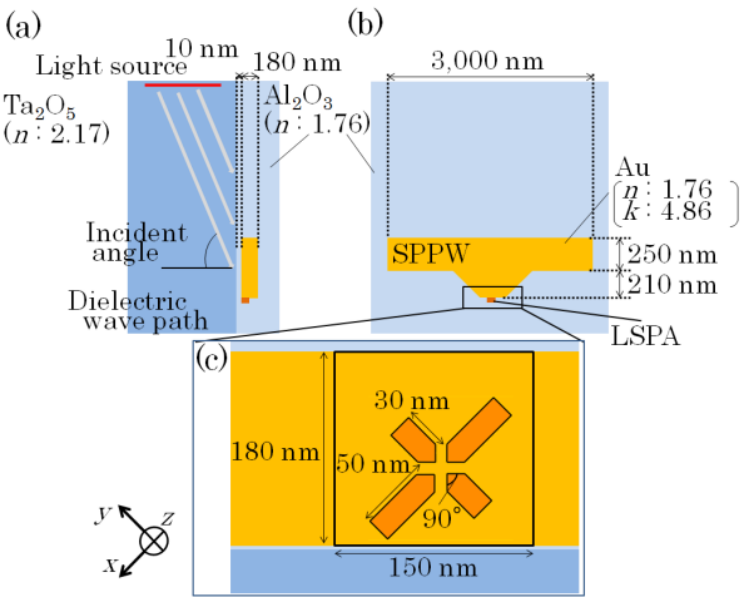

(a) Side view (b) Front view (c) Bottom view

Fig. 6 Computational model which combined LSPA and SPPW. 
$\left(E_{\mathrm{y}} / E_{\mathrm{x}}=0.96\right)$, and the phase difference between $E_{\mathrm{x}}$ and $E_{\mathrm{y}}$ was 90 degrees. Furthermore, the degree of circularity $C$ and power intensity of electric field $I^{6)}$ were evaluated as the confined circularly polarized light. The $C$ map surrounding the LSPA is shown in Fig. 8. These values were defined as follows:

$$
C=\frac{<2 E_{x} E_{y} \sin \theta>}{<E_{x}{ }^{2}>+<E_{y}{ }^{2}>+<E_{z}{ }^{2}>},
$$

where $<>$ corresponds to taking a time average, $\theta$ is the phase difference between $x$ and $y$ components of electric field,

$$
I=<E_{x}^{2}>+<E_{y}^{2}>+<E_{z}^{2}>.
$$

$C$ and $I$ were calculated at $1 \mathrm{~nm}$ below the LSPA, which was placed on the bottom of the SPPW after 70 fs from laser irradiation. A circularly polarized light was confined to $5 \mathrm{~nm}$ in diameter. The $C$ value of the confined area was kept at 0.8 as shown in Fig. 8. This result means that the propagated surface plasmon polariton along with the SPPW was transformed into the localized surface plasmon at the LSPA. However, power intensity $I$ is concentrated at the edge of the plasmon antenna. We need to have a peak at the center of the cross antennas. But it is expected to be achieved to concentrate the power intensity $I$ at center by adapting particulate media into the computational model, because it was revealed that the intensity $I$ can be focused into the particle placed on the bottom of a LSPA center in the previous study ${ }^{4}$.

LSPA is effective for high density recording. The SPPW is useful for high efficiency energy propagation. It was revealed that the combination of the LSPA with the SPPW created a localized circularly polarized light. We are going to study more details with recording media for our future research.

\section{Conclusion}

A new antenna array combining LSPA and SPPW was studied. It is confirmed that the cross antenna placed under a metal sheet can create a localized circular polarization by indirect energy from a metal layer. In changing the length and position of a rectangular shaped plasmon antenna attached on the bottom of SPPW, the phase of the electric field generated by the antenna can be shifted. It is demonstrated that the combined model of LSPA for high density magnetic recording and SPPW for high efficiency energy propagation can generate a circularly polarized light at the center of the LSPA. Circular polarization can be confined to $5 \mathrm{~nm}$ in diameter. Additionally, the value of $C$ within the confined area can be kept at 0.8 . This technique is expected to be useful for ultra-fast, high density, and energy efficient magnetic recording.

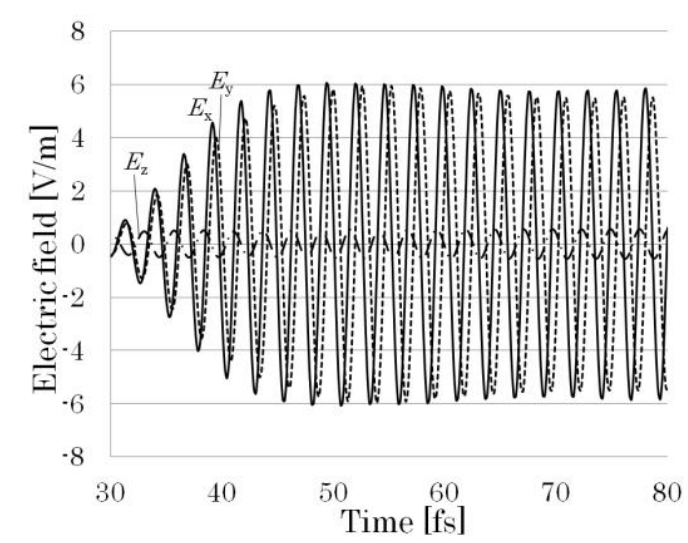

Fig. 7 Time responses of electric field $x, y$, and $z$ components $\left(E_{\mathrm{x}}, E_{\mathrm{y}}\right.$ and $\left.E_{\mathrm{z}}\right)$ at the center of the cross antenna placed on the bottom of the SPPW.

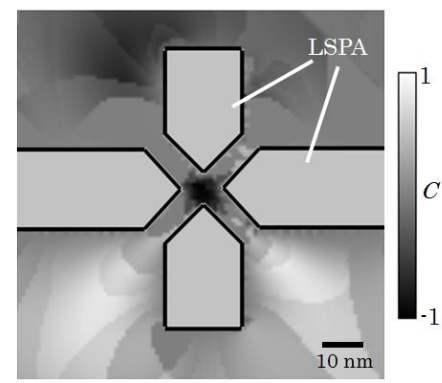

Fig. 8 The degree of circularity $C$, defined by equation (1) on the bottom surface of LSPA as shown Fig. 6 (c).

Acknowledgements This work is partially supported by a Grant-in-Aid for Scientific Research (C), No. 23560413, a Grant of Storage Research Consortium, and a Grant of Nihon University Strategic Projects for Academic Research.

\section{References}

1) C. D. Stanciu, F. Hansteen, A. V. Kimel, A. Kirilyuk, A. Tsukamoto, A. Itoh, and Th. Rasing: Phys. Rev. Lett., 99, 047601 (2007).

2) K. Nakagawa, Y. Ashizawa, S. Ohnuki, A. Itoh, and A. Tsukamoto: J. Appl. Phys., 109, 07B735 (2011)

3) T. Ota, Y. Ashizawa, K. Nakagawa, S. Ohnuki, H. Iwamatsu, A.Tsukamoto, and A. Itoh: J. Magn. Soc. Jpn., 36, 66 (2012).

4) H. Iwamatsu, T. Okada, S. Kishimoto, S. Ohnuki, Y. Ashizawa, and K. Nakagawa: Tech. Rep., IEE Japan, EMT-10-123,121 (2010).

5) Y. Ashizawa, T. Ota, and K. Nakagawa, IEEE Int. Magnetics Conf., CC-06, 2012.

6) P. Biagioni, J. S. Huang, L. Duò, M. Finazzi, and B. Hecht: Phys. Rev. Lett., 102, 256801 (2009).

7) Gerrit Mur: IEEE Trans. Microw. Theory Tech., MTT-29, 1073 (1981).

Received Oct. 19, 2012; Revised Dec. 06, 2012;

Accepted Jan. 18, 2013 